\title{
Brewster effect in metafilms composed of bi-anisotropic split-ring resonators
}

\author{
Yasuhiro Tamayama ${ }^{1, *}$ \\ ${ }^{1}$ Department of Electrical Engineering, Nagaoka University of Technology, \\ 1603-1 Kamitomioka, Nagaoka, Niigata 940-2188, Japan
}

compiled: June 27, 2021

\begin{abstract}
The Brewster effect is extended to single-layer metafilms. In contrast to bulk media, the Brewster effect in metafilms can be realized by tailoring the radiation pattern of a distribution of meta-atoms rather than the effective medium parameters. A metafilm composed of bi-anisotropic split-ring resonators is designed based on the theory, and its characteristics are numerically analyzed. The simulation demonstrates that there exists a condition for which the polarization of the reflected wave becomes independent of the incident polarization at a particular angle of incidence.

OCIS codes: (160.3918) Metamaterials; (260.2110) Electromagnetic optics; (230.5440) Polarizationselective devices.

http://dx.doi.org/10.1364/XX.99.099999
\end{abstract}

The Brewster effect arises in connection with the laws of reflection and refraction of electromagnetic waves at an interface between two different media. When an electromagnetic wave is incident on the interface at a particular angle of incidence, which is called the Brewster angle, the polarization of the reflected wave becomes independent of the incident polarization. It can alternatively be considered an antireflection phenomenon because the reflected wave vanishes for a certain polarization of the incident wave. This effect is applied, for example, to generate polarized light from unpolarized light and to suppress reflection losses at intracavity elements.

The antireflection condition is only satisfied for transverse-magnetic (TM) waves (horizontally polarized waves) in naturally occurring media because such materials do not exhibit a magnetic response in high frequency regions, i.e., microwave, terahertz, and optical regions. It was not until metamaterials [1 5] were developed that the antireflection effect was experimentally observed for non-TM polarizations [6, 7]. As far, the Brewster effect has been studied in magnetic media 8 11], anisotropic media 12 15], chiral (bi-isotropic) media 16 18], bi-anisotropic media [19], and plasmonic media 20 22].

The physical meaning of the Brewster effect is based on the radiation patterns of the electric dipole moment and magnetic moment induced in the material [9]. For simplicity, a plane electromagnetic wave is assumed to be incident from a vacuum onto a pure dielectric. When a

* Corresponding author: tamayama@vos.nagaokaut.ac.jp transverse-electric (TE) wave (vertically polarized wave) is incident on the medium, the direction of vibration of the induced electric dipoles is perpendicular to the plane of incidence. The radiation pattern in the incident plane is isotropic, so that the reflected wave does not vanish for any angle of incidence. On the other hand, for horizontal incident polarization, the direction of oscillation of the electric dipoles is in the incident plane and the radiation pattern in the incident plane has a null in the direction of vibration. If the propagation direction of the reflected wave coincides with this null direction, the horizontally polarized reflected wave vanishes. Therefore, the reflected wave becomes vertically polarized regardless of the incident polarization for this propagation direction.

Although the Brewster effect is thought of as a phenomenon that occurs at an interface between two bulk media, the principle can be extended to single-layer metafilms. In this study, it is shown that the Brewster effect, in which the polarization of the reflected wave becomes independent of the incident polarization at a particular angle of incidence [16-19], can be achieved in metafilms by tailoring the directions of the electric dipole moment and magnetic moment in the constituent meta-atoms and by suitably arranging the spatial distribution of those meta-atoms. The method is developed through a theoretical analysis of the polarization of the radiated wave from an array of bi-anisotropic split-ring resonators (SRRs) 23 26]. The reflection and transmission characteristics of the metafilm are analyzed using a finite-difference time-domain (FDTD) method. The results demonstrate that there exists a condition for which the reflected wave is vertically polarized independent of 

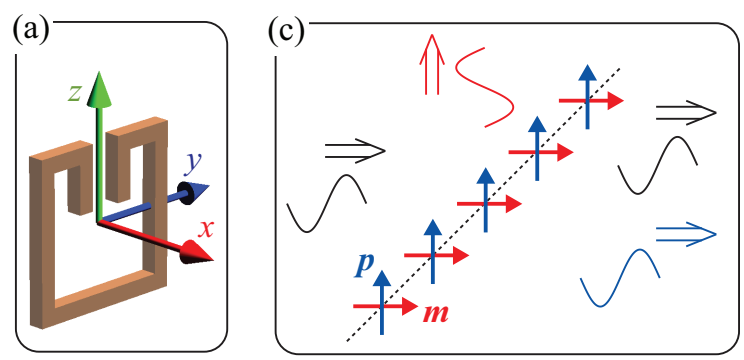

(b)

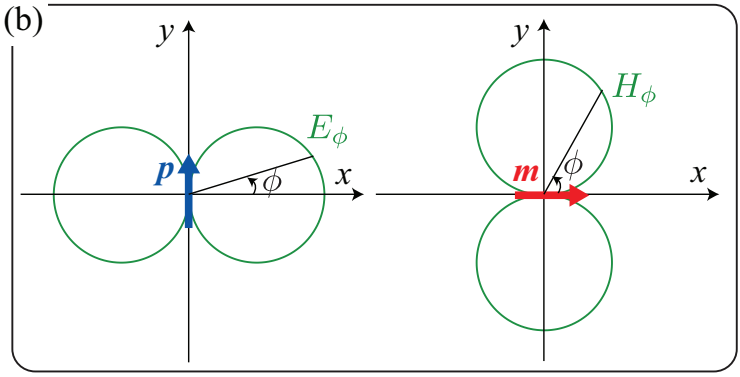

Fig. 1. (a) Geometry of the bi-anisotropic SRR and of the coordinate system. (b) Radiation patterns in the $x y$ plane of $\boldsymbol{p}$ and $\boldsymbol{m}$ induced in the SRR. (c) Example of the relationship among the SRRs and the propagation directions of the incident, reflected, and transmitted waves to achieve the Brewster effect in the metafilm. The incident wave is shown in black and the radiation from $\boldsymbol{p}(\boldsymbol{m})$ is shown in blue (red).

the incident polarization.

To develop a method for achieving the Brewster effect in metafilms of bi-anisotropic SRRs, consider the radiation pattern of the single SRR in Fig.1(a). The electric dipole moment $\boldsymbol{p}$ is in the $y$ direction and the magnetic moment $\boldsymbol{m}$ is in the $x$ direction, assuming that the incident electromagnetic waves only excite circular currents in the SRR. The radiation patterns in the $x y$ plane of $\boldsymbol{p}$ and $\boldsymbol{m}$ are sketched in Fig.1(b). The radiation pattern of $\boldsymbol{p}(\boldsymbol{m})$ has maxima (nulls) at $\phi=0^{\circ}$ and $180^{\circ}$ and nulls (maxima) at $\phi=90^{\circ}$ and $270^{\circ}$. The polarization direction of the wave radiated from $\boldsymbol{p}$ is in the $x y$ plane (horizontally polarized) and that from $\boldsymbol{m}$ is perpendicular to the $x y$ plane (vertically polarized). Thus, the polarization of the radiated wave in the directions of $\phi=0^{\circ}$ and $180^{\circ}\left(\phi=90^{\circ}\right.$ and $\left.270^{\circ}\right)$ is always horizontal (vertical).

The radiation pattern of the metafilm is determined by the product of the radiation pattern of the constituent meta-atom and the array factor [27], which is determined by the phase difference between waves radiated from neighboring meta-atoms. If the array factor vanishes for all angles except $\phi=0^{\circ}$ and $180^{\circ}\left(90^{\circ}\right.$ and $\left.270^{\circ}\right)$, the radiated wave is horizontally (vertically) polarized. Therefore, when the propagation direction of the reflected wave is equal to $\phi=0^{\circ}$ or $180^{\circ}\left(90^{\circ}\right.$ or $\left.270^{\circ}\right)$ as a result of tailoring the configuration of the SRRs and the propagation direction of the incident wave, the reflected wave becomes horizontally (vertically) polarized

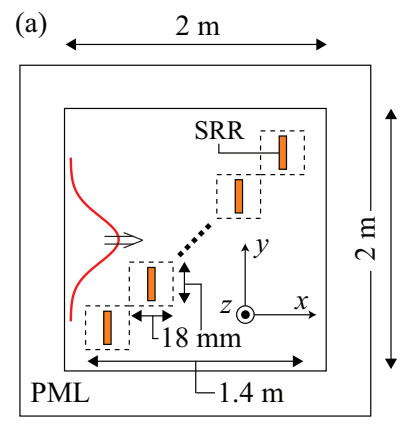

(b)

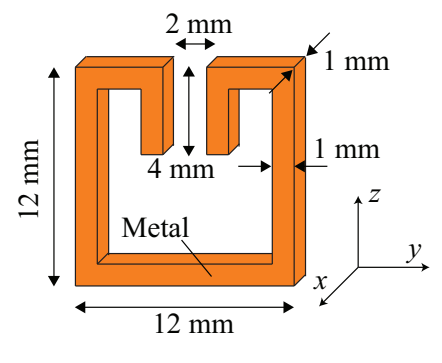

Fig. 2. (a) Schematic of the simulation system. (b) Geometrical parameters of the bi-anisotropic SRR. The unit cell of the metafilm has dimensions of $18 \mathrm{~mm} \times 18 \mathrm{~mm} \times 18 \mathrm{~mm}$. The conductivity of the metal is taken to be $5.8 \times 10^{7} \mathrm{~S} / \mathrm{m}$.

independent of the incident polarization.

To realize this Brewster condition, the SRRs should be arranged, for example, periodically in the $z$ direction and in the direction $\phi=45^{\circ}$, as depicted in Fig.11(c). When the electromagnetic wave is incident from $\phi=180^{\circ}$, the metafilm radiates electromagnetic waves in the directions $\phi=0^{\circ}$ and $90^{\circ}$, as determined by the phase difference between radiation from neighboring SRRs (i.e., conservation of linear momentum in the direction parallel to the surface of the metafilm). That is, the array factor for this case vanishes for all angles except $\phi=0^{\circ}$ and $90^{\circ}$. If the incident wave is horizontally polarized, the metafilm is excited and a vertically polarized wave is reflected in the direction $\phi=90^{\circ}$, as indicated by the red wave in Fig.1(c). On the other hand, for vertical incident polarization, the metafilm is not excited and the reflection vanishes. Thus, the reflected wave is vertically polarized independent of the incident polarization. (When the electromagnetic wave is incident from the direction $\phi=90^{\circ}$, the reflected wave is horizontally polarized independent of the incident polarization according to the above theory. However, that does not occur for a metafilm composed of actual bi-anisotropic SRRs, as explained later.)

The reflection and transmission characteristics of the metafilm are numerically analyzed using an FDTD method to demonstrate the Brewster effect in the metafilm. The simulation system is sketched in Fig.2(a). The simulation space has dimensions of $2 \mathrm{~m} \times 2 \mathrm{~m} \times$ $18 \mathrm{~mm}$ and is discretized into uniform cubes with dimensions of $1 \mathrm{~mm} \times 1 \mathrm{~mm} \times 1 \mathrm{~mm}$. Perfectly matched layer (PML) boundary conditions are applied in the $x$ and $y$ directions, while periodic boundary conditions are applied in the $z$ direction. The bi-anisotropic SRRs shown in Fig.2(b) are periodically distributed at $\phi=45^{\circ}$ to compose the metafilm. A Gaussian beam with a spot width of $40 \mathrm{~cm}$ and a Rayleigh range of $1.3 \mathrm{~m}$ is incident from the left onto the metafilm.

Figure 3 plots the reflection and transmission spectra of the metafilm when an electromagnetic wave is incident 

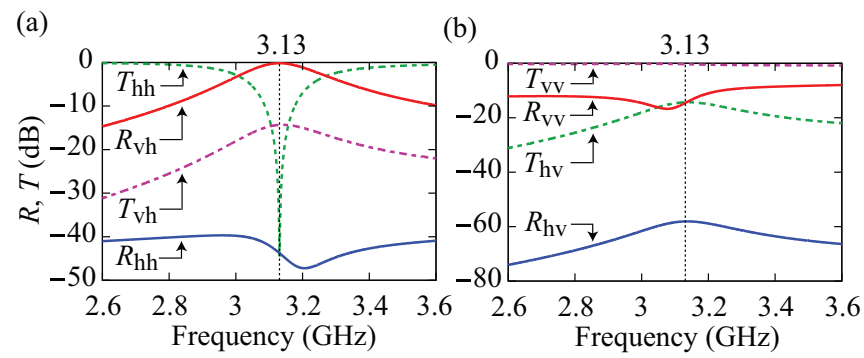

Fig. 3. Reflection and transmission spectra of the metafilm for (a) horizontal and (b) vertical incident polarization.

from $\phi=180^{\circ}$. The first subscript indicates the polarization of the reflected/transmitted wave, while the second subscript indicates the polarization of the incident wave, with $\mathrm{v}$ and $\mathrm{h}$ referring to vertical and horizontal polarizations, respectively. The reflectance (transmittance) is calculated from the far-field pattern in the direction $\phi=90^{\circ}\left(\phi=0^{\circ}\right)$ of the reflected (transmitted) wave.

Figure 3(a) graphs the spectra for horizontal incident polarization. At the resonant frequency of the SRR, namely $3.13 \mathrm{GHz}, R_{\mathrm{vh}}$ has a maximum value and $T_{\mathrm{hh}}$ has a minimum value, for the following reason. The reflected wave is composed solely of the radiated wave from the metafilm. The amplitude of $\boldsymbol{m}$ is largest at the resonant frequency, and thus the reflectance is maximum. On the other hand, the transmitted wave is composed of a superposition of the incident and radiated waves. Since the response of $\boldsymbol{p}$ is a resonant (Lorentz) type, the incident wave and the radiation from $\boldsymbol{p}$ cancel each other at the resonant frequency; thus the transmittance becomes a minimum. The other components, $T_{\mathrm{vh}}$ and $R_{\mathrm{hh}}$, should vanish in the theory but actually have nonzero values due to minor responses of the metafilm such as multiple scattering and a radiation from an electric dipole moment $\boldsymbol{p}^{\prime}$ excited by the electric field in the $z$ direction, which are not taken into account in the theory. (The response of $\boldsymbol{p}^{\prime}$ is non-resonant in this frequency range. The radiation pattern in the $x y$ plane of $\boldsymbol{p}^{\prime}$ is isotropic and the radiation is vertically polarized.) The ratio $R_{\mathrm{vh}} / R_{\mathrm{hh}}$ is larger than $10^{4}$ at the resonant frequency; hence the reflected wave is essentially vertically polarized. Note that $R_{\mathrm{vh}} / R_{\mathrm{hh}}$ is large even in the near off-resonant region. The polarization of the reflected wave is vertical whenever the principal component of the reflected wave is from $\boldsymbol{m}$.

Figure 3(b) shows the reflection and transmission spectra for vertical incident polarization. The interaction between the incident wave and the metafilm is weak because the vertically polarized incident wave cannot directly induce $\boldsymbol{p}$ and $\boldsymbol{m}$. Thus $T_{\mathrm{vv}}$ is nearly $0 \mathrm{~dB}$ at every frequency. Since a vertically polarized incident wave can directly induce $\boldsymbol{p}^{\prime}, R_{\mathrm{vv}}$ is comparable to or larger than the minor components $T_{\mathrm{hv}}$ and $R_{\mathrm{hv}}$. The ratio $R_{\mathrm{vv}} / R_{\mathrm{hv}}$ is larger than $10^{4}$ at the resonant frequency and remains

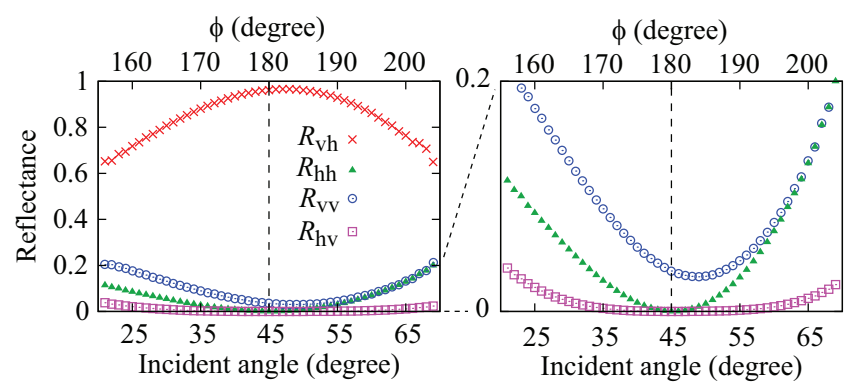

Fig. 4. Reflectance of the metafilm as a function of the incident angle for the frequency of $3.13 \mathrm{GHz}$. The angle $\phi$ is the direction from which the electromagnetic wave is incident on the metafilm. The right panel is a magnification of the left panel in the region of low reflectance.

large in the off-resonant region. Therefore, the polarization of the reflected wave is also vertical for vertical incident polarization.

Figure 4 shows the dependence of the reflectance of the metafilm on the incident angle for the frequency of $3.13 \mathrm{GHz} . R_{\mathrm{hh}}$ and $R_{\mathrm{hv}}$ vanish at the incident angle of $45^{\circ}$, which reflects the radiation pattern of $\boldsymbol{p}$ shown in Fig.1(b). On the other hand, $R_{\mathrm{vh}}$ and $R_{\mathrm{vv}}$ have nonzero values at the incident angle of $45^{\circ}$. This implies that the reflected wave is vertically polarized regardless of the incident polarization at the incident angle of $45^{\circ}$. For all angles of incidence except $45^{\circ}$, all of the reflectances have nonzero values and such a polarizing effect does not occur. Therefore, the incident angle of $45^{\circ}$ is confirmed to be the Brewster angle of the metafilm.

If $\boldsymbol{p}^{\prime}$ were not induced in the SRR, the Brewster effect would occur when the plane wave is incident from the direction $\phi=90^{\circ}$. The reflected wave would then become horizontally polarized. However, that does not occur due to the electric dipole moment $\boldsymbol{p}^{\prime}$ that arises because the SRR is made of an electric conductor, and a small vertical polarization component occurs in the reflected wave for vertical incident polarization. If the bi-anisotropic SRR is instead made of a magnetic conductor, then $\boldsymbol{p}^{\prime}$ would vanish and the Brewster effect would occur for the electromagnetic wave incident from $\phi=90^{\circ}$. (Of course, the Brewster effect does not occur for the electromagnetic wave incident from $\phi=180^{\circ}$ in this case.)

The Brewster effect in metafilms and that in bulk media have similar characteristics in the reflection Jones matrix as well as in the physical meaning. The reflection Jones matrix $M_{\mathrm{r}}$ is defined by the relation $\left[E_{\mathrm{rv}} E_{\mathrm{rh}}\right]^{t}=$ $M_{\mathrm{r}}\left[\begin{array}{ll}E_{\mathrm{iv}} & E_{\mathrm{ih}}\end{array}\right]^{t}=\left[\begin{array}{ll}\boldsymbol{r}_{1} & \boldsymbol{r}_{2}\end{array}\right]^{t}\left[\begin{array}{ll}E_{\mathrm{iv}} & E_{\mathrm{ih}}\end{array}\right]^{t}$ where $E_{\mathrm{r}}\left(E_{\mathrm{i}}\right)$ is the electric field amplitude of the reflected (incident) wave, the second subscript indicates the polarization, and $t$ denotes the transpose. The column vectors $\boldsymbol{r}_{1}$ and $\boldsymbol{r}_{2}$ that compose $M_{\mathrm{r}}$ are respectively written as $\boldsymbol{r}_{1}=\left[\begin{array}{ll}r_{\mathrm{vv}} & r_{\mathrm{vh}}\end{array}\right]^{t}$ and $\boldsymbol{r}_{2}=\left[\begin{array}{ll}r_{\mathrm{hv}} & r_{\mathrm{hh}}\end{array}\right]^{t}$ where $r_{\alpha \beta}=\sqrt{R_{\alpha \beta}} \exp \left[\mathrm{i} \arg \left(r_{\alpha \beta}\right)\right]$ $(\alpha, \beta=\mathrm{v}, \mathrm{h})$ is a complex reflection coefficient. The 
Brewster condition in bulk media is given by the vanishing of the determinant of the reflection Jones matrix [1619]. For the present metafilm, it is found from Fig.4 that $\boldsymbol{r}_{2}$ is zero vector at the Brewster angle; therefore, the determinant of the reflection Jones matrix also vanishes at the Brewster angle. Note that the reflected wave vanishes at the Brewster angle for the incident polarization that satisfies $E_{\mathrm{iv}} / E_{\mathrm{ih}}=-r_{\mathrm{vh}} / r_{\mathrm{vv}}$.

In conclusion, an extension of the Brewster effect to metafilms composed of bi-anisotropic SRRs has been studied by a theoretical analysis and by full-wave simulations. Consideration of the radiation patterns of $\boldsymbol{p}$ and $\boldsymbol{m}$ indicates that the polarization of the reflected wave is independent of the incident polarization when the SRRs (whose axes are in the direction $\phi=0^{\circ}$ ) are periodically arranged in the $z$ direction and in the direction $\phi=45^{\circ}$ if the electromagnetic wave is incident on the metafilm at the incident angle of $45^{\circ}$. The reflection and transmission characteristics of the metafilm have been numerically analyzed using an FDTD method. The simulation demonstrates that the polarization of the reflected wave is vertical, independent of the incident polarization when the electromagnetic wave is incident from the direction $\phi=180^{\circ}$ onto the metafilm. The determinant of the reflection Jones matrix at the Brewster angle has been evaluated based on the numerical analysis, and the mathematical formulation of the Brewster effect in metafilms is found to be the same as that in bulk media. The Brewster effect in the present metafilm can be applied to reflective optical elements that have combined characteristics of polarizer and polarization converter. Such Brewster effect has not been observed for bulk media. This study demonstrates that the Brewster effect can be designed in metafilms by tailoring a radiation pattern of constituent meta-atoms and a spatial distribution of those meta-atoms rather than by varying effective medium parameters. This concept would enable us to create useful optical elements that cannot be achieved by the method based on effective medium parameters.

This study was supported by a Grant-in-Aid for Scientific Research on Innovative Areas (No. 22109004) from the Ministry of Education, Culture, Sports, Science, and Technology of Japan.

\section{References}

[1] D. R. Smith, J. B. Pendry, and M. C. K. Wiltshire, Science 305, 788 (2004).

[2] B. Wang, J. Zhou, T. Koschny, M. Kafesaki, and C. M. Soukoulis, J. Opt. A: Pure Appl. Opt. 11, 114003 (2009).
[3] H. Tao, W. J. Padilla, X. Zhang, and R. D. Averitt, IEEE J. Sel. Top. Quantum Electron. 17, 92 (2011).

[4] C. M. Soukoulis and M. Wegener, Nature Photon. 5, 523 (2011).

[5] N. I. Zheludev and Y. S. Kivshar, Nature Mater. 11, 917 (2012).

[6] Y. Tamayama, T. Nakanishi, K. Sugiyama, and M. Kitano, Phys. Rev. B 73, 193104 (2006).

[7] R. Watanabe, M. Iwanaga, and T. Ishihara, Phys. Stat. Sol. (b) 245, 2696 (2008).

[8] W. T. Doyle, Am. J. Phys. 48, 643 (1980).

[9] G. P. Sastry and S. Chakrabarty, Eur. J. Phys. 8, 125 (1987).

[10] J. Futterman, Am. J. Phys. 63, 471 (1995).

[11] C. Fu, Z. M. Zhang, and P. N. First, Appl. Opt. 44, 3716 (2005).

[12] T. M. Grzegorczyk, Z. M. Thomas, and J. A. Kong, Appl. Phys. Lett. 86, 251909 (2005).

[13] T. Tanaka, A. Ishikawa, and S. Kawata, Phys. Rev. B 73, 125423 (2006).

[14] N.-H. Shen, Q. Wang, J. Chen, Y.-X. Fan, J. Ding, and H.-T. Wang, J. Opt. Soc. Am B 23, 904 (2006).

[15] W. Shu, Z. Ren, H. Luo, and F. Li, Appl. Phys. A 87, 297 (2007).

[16] S. Bassiri, C. H. Papas, and N. Engheta, J. Opt. Soc. Am. A 5, 1450 (1988).

[17] A. Lakhtakia, Opt. News 15, 14 (1989).

[18] Y. Tamayama, T. Nakanishi, K. Sugiyama, and M. Kitano, Opt. Express 16, 20869 (2008).

[19] A. Lakhtakia, Optik (Stuttgart) 90, 184 (1992).

[20] A. Alù, G. D'Aguanno, N. Mattiucci, and M. J. Bloemer, Phys. Rev. Lett. 106, 123902 (2011).

[21] N. Aközbek, N. Mattiucci, D. de Ceglia, R. Trimm, A. Alù, G. D'Aguanno, M. A. Vincenti, M. Scalora, and M. J. Bloemer, Phys. Rev. B 85, 205430 (2012).

[22] C. Argyropoulos, G. D'Aguanno, N. Mattiucci, N. Akozbek, M. J. Bloemer, and A. Alù, Phys. Rev. B 85, 024304 (2012).

[23] N. Katsarakis, T. Koschny, M. Kafesaki, E. N. Economou, and C. M. Soukoulis, Appl. Phys. Lett. 84, 2943 (2004).

[24] S. Linden, C. Enkrich, M. Wegener, J. Zhou, T. Koschny, and C. M. Soukoulisz, Science 306, 1531 (2004).

[25] C. Rockstuhl, T. Zentgraf, H. Guo, N. Liu, C. Etrich, I. Loa, K. Syassen, J. Kuhl, F. Lederer, and H. Giessen, Appl. Phys. B 84, 219 (2006).

[26] H. Tao, A. C. Strikwerda, K. Fan, W. J. Padilla, X. Zhang, and R. D. Averitt, Phys. Rev. Lett. 103, 147401 (2009).

[27] R. C. Johnson, ed., Antenna Engineering Handbook, 3rd ed. (McGrow-Hill, New York, NY, 1992). 